\title{
3-D Reconstruction of Image Structures Based-on Double Optical Paths Microscopy
}

\author{
Qiong Cai, BinYan, Hua Xiang, Junming Chang, Guangyi Zou \\ School of Mathematics \& computer science \\ Jianghan University
}

\begin{abstract}
This paper presents a method reconstructing 3-d image structures with data collected from double optical paths microscopy. A color image gathered is divided into two monochromatic images which respectively represent right and left vision data by color filter. Then the image structures are extracted by median filter and calculated with Sobel operator or Canny operator. Pixels between the right and left images are corresponded by stereo matching. Finally 3-d real-time reconstruction of micro image is realized.
\end{abstract}

Keywords-Binocular vision, 3-d reconstruction, Stereo matching

\section{INTRODUCTION}

It is normal that date is extracted by single CCD, observed by binocular naked eyes in the microscope application. Researchers search those stereo reconstruction methods based on this application. References [1] derives a precise expression for the bias in the depth estimate, based on the continuous (differentiable) version of structure from motion (SfM), How monocular image cues can be combined with triangulation cues to build a photo-realistic model of a scene given only a few images-even ones taken from very different viewpoints or with little overlap is shown in references [2]. Geometric optimization-based algorithms for simultaneous 3-D shape (depth) and motion (linear and angular velocities) recovery that minimizes the squared distance between the observed and predicted optical flow measurements is shown in reference [3].A comparison of Three 3-D Facial Reconstruction Approaches is given in the literature [4].

Those data which is kept by computer is all two dimensional data. Consequently, the formed stereo is stereo model.

Those applications which is currently less used in the microscope application instantly and simultaneously is collected by double optical paths, allocate double path is applied to collect two CCD from left-right eyepiece, form static images which are 2 paths with different visual. Two static images are colored synthesizd[5] different visual images can be formed by double color ocular, stereo synthesis by human brain can be formed. But how to make the image in computer match with the image by observer's brain, this is the focus point of this research.

Human's visual habit is taken into consideration, the original information from 2 optical path branches is obtained instantly and operators are apperceived the stereo microobject storage directly in the computer by their eyes, the real "what you saw is what you get" is realized, characteristics of all kinds of samples can be picked out and reappear the configuration of stereo object.

This research can be considered as it realizes the original stereo is obtained simultaneously and afterwards analysis. Consequently, this image analysis system is more suitable in samples' collection and analysis of biology and medical science fields. Any object's stereo profile can be confirmed by using of isolated optical path's digitalized 3-d reconstruction system of 3-d micro information, and 3-d coordinate can be obtained at any point.

\section{TeChNicAl SpecificAtion}

For the collected double optical path images is separated into two left-right optical paths images, edge line of construction can be manifested by every extracted image, and then left-right light is synthesized into new 3-d construction to realize stereo reconstruction.

\section{A. Color Isolation}

Original color image $P$ is $X \times Y$ image which is obtained by computer in 2-d coordinate, $(x, y)$ is a random pixel which color parameter is $z$, it is marked as $(x, y, z) . z$ is obtained by red, green, blue which are 8 bits colors stack together, it is marked as $z=\left(z_{1}, z_{2}, z_{3}\right)$,

whole image can be presented as following:

$$
\begin{aligned}
& P_{I}=\left\{I(x, y, z) \mid z=z_{1}+z_{2}+z_{3}, x: 0-X,\right. \\
& \left.y: 0-Y, z_{1}: 0-255, z_{2}: 0-255, z_{3}:-255\right\}
\end{aligned}
$$

Red, green and blue paths separately are:

$$
\begin{aligned}
& P_{R}=\left\{I\left(x, y, z_{1}\right) \mid x: 0-X, y: 0-Y, z_{1}: 0-255\right\} \\
& P_{G}=\left\{I\left(x, y, z_{2}\right) \mid x: 0-X, y: 0-Y, z_{2}: 0-255\right\} \\
& P_{B}=\left\{I\left(x, y, z_{3}\right) \mid x: 0-X, y: 0-Y, z_{3}: 0-255\right\}
\end{aligned}
$$

\section{B. Structure Extraction}

Edge is the border of 2 images area with different grey level. Edge point is chosen as primitive for later match. The grey levels changed smoothly go along with the edge's trend, while grey level which is vertical with the edge trend is changed fiercely. Grey level value on edge is the result of discontinuity, this kind of discontinuity can be checked out easily by the way of getting the derivative. Edge point is corresponded with the max value of first order derivative 
range; this point is also corresponded with second order derivative's zero crossing. So it is a workable measure to use first order differential operator and second order differential operator to make the edge check.

The image edge can be obtained by a clear edge image (Refer to Fig 1.) by median filtering and Sober arithmetic operators or median filter add Canny arithmetic operators. (Refer to Fig 2., 3.)

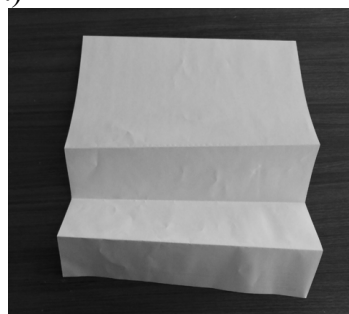

Figure 1. original image

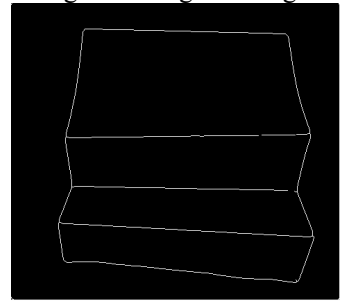

Figure 2. median and sober arithmetic operators extract

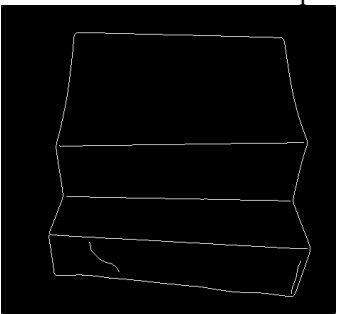

Figure 3. median filter add canny arithmetic operators extrac

\section{Stereo Matching Issue}

Imaging point of a same object point in 2 microscopes is called symmetry point, a certain point in image is given in the essence of stereo match[6] to search corresponding point in another image, to make these 2 points are the projection of a same object point. It is a precondition to research matching algorithm by reasonable selection and high precision extract chosen matching characteristic, according to parallax theory to confirm the depth of 3-d object need to first confirm corresponding image point on the stereo image, which is to confirm corresponding image point by image characteristic, this is an issue about matching characteristic selection. Currently, there isn't a common applied theory can be used in matching characteristic extraction, this leads to diversity of matching characteristic in 3-d visual research. A favorable matching characteristic should have characteristics such as distinction, fixity, stability, uniqueness and ability of solving the ambiguity matching. Therefore, which characteristics as matching characteristics need to be confirmed firstly, and then how to extract those characteristics and match them up to get the parallax information, these two questions is connected and affected with each other.
The method of edge line matching[7] is applied in this research. Discrete Fourier transform [8] is applied to obtain the approximate description of edge line. A point $\left(x_{1}, y_{1}\right)$ is taken on the edge line, the coordinate sequence $\left(x_{i}, y_{i}\right), i=1,2 \cdots n$. is read along with a certain direction, $P(i)=x_{i}, Q(i)=y_{i}$ is assumed, and then edge line is expressed as:

$$
L(k)=[P(k), Q(k)]
$$

$X Y$ plane is coincided with $P Q$ plane; $X Y$ coordinate is coincided with PO coordinate, edge line is expressed as complex number sequence:

$$
L(k)=P(k)+i Q(k), k=1,2, \cdots, n
$$

Fourier transform is taken as discrete:

$$
L(\lambda)=\frac{1}{n+1} \sum_{k=1}^{n} L(k) e^{\frac{-2 \pi k \lambda i}{n+1}}, \lambda=1,2, \cdots, n
$$

$L(\lambda)$ in formula $(7)$ is the approximate description of edge line. We can confirm from this formula if the edge line of left and right images are matched, after edge line is obtained, these 3 -d reconstruction image of these edge lines can be obtained by method 2.3.These lines are combined together to get whole reconstruction 3-d image.

\section{3-d Reconstruction}

Assuming double paths collect 2 CCD which 3-d microscope's 2 drawtubes optical paths are vertical with each other, if binocular optical center's axle base is $d$, ocular focal length is $f$. Origin of microscope coordinate system on the point of intersection of optical axis of microscope and plane, left right image plane is in front of optical center $f$,random point $P$ is marked as $P\left(x_{p}, y_{p}, z_{p}\right)$ in the left microscope coordinate system, corresponding coordinate in the left and right figure is $X_{l}\left(x_{l}, y_{l}\right)$ and $X_{r}\left(x_{r}, y_{r}\right)$,because two oculars are on the same plane, the image coordinate of point $P$ is same with y coordinate, namely $y_{l}=y_{r}$.Following formula from the relationship of triangle geometric is obtained.

$$
x_{l}=f \frac{x_{p}}{z_{p}}, x_{r}=f \frac{\left(x_{p}-d\right)}{z_{p}}, y_{l}=y_{r}=f \frac{y_{p}}{z_{p}}
$$

Definition of parallax is a certain point which is the corresponding position shift in two different images:

$$
\Delta x=\left|x_{l}-x_{r}\right|=\frac{f \cdot d}{z_{p}}
$$


According to it, coordinate of a certain point $\mathrm{P}$ in left ocular coordinate system can be calculated out:

$$
x_{p}=\frac{d x_{l}}{\Delta x}, y_{p}=\frac{d f}{\Delta x}, z_{p}=\frac{d f}{\Delta x}
$$

Consequently, as long as corresponding point of a certain point in left and right imaging plane can be found, and the parameter $d$ and $f$ from microscope calibration is obtained to realize $3-\mathrm{d}$ reconstruction.

A inclined curve is put under the microscope, the coordinate of left-right imaging plane points on the curve can be read from coordinate $y$ according to arithmetic procession in little endian format, 2 read sequence data are $S_{r 1}\left(x_{r i}, y_{r i} \mid i=0,1, \ldots n\right)$ and $S_{l l}\left(x_{l i}, y_{l i} \mid i=0,1, \ldots n\right)$, $\left(x_{i}, y_{i}\right)$ is the coordinate of number $i$, point $\mathrm{n}$ in the left image and it's corresponding point in right image can be found, sequence points on the curve can be calculated out a $3-d$ coordinate from formula (10), and then 3-d reconstruction image of this curve can be obtained.

\section{LABORATORY RESEARCH}

Refer to Figure 4. and 5., construction of Left-right path images are extracted, two paths' construction data sequence of Figure 4., 5. is obtained by horizontal scanning.

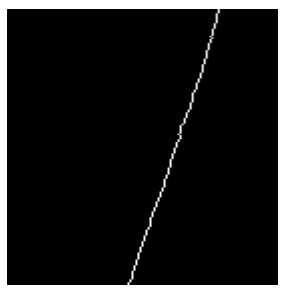

Figure 4. left image

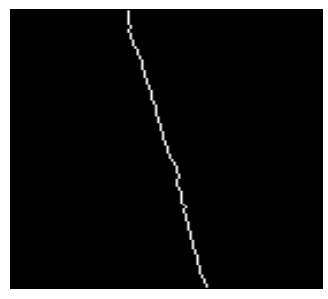

Figure 5. right image
By formula (3), all the 3-d coordinate on this curve can be calculated out, then 3 -d reconstruction can be obtained on this curve (Fig.6.).

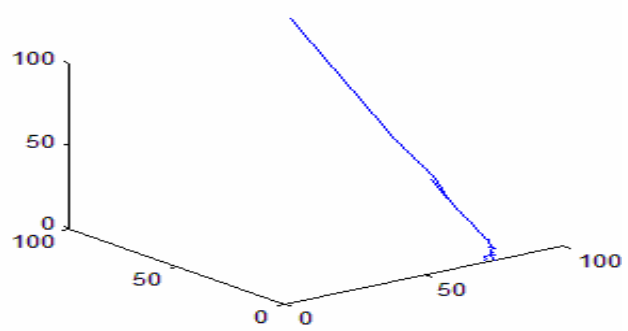

Figure 6. the 3-d image after reconstruction lines.

\section{SUMMARIES}

Firstly, principle of optical isolation is used in this research to obtain double optical paths' left and right images. Image edge can be obtained by a clear edge image by median filtering and Sober arithmetic operators or median filter add Canny arithmetic operators, and then method of edge lines matching is applied to find the matching point of left right image, finally the $3-\mathrm{d}$ reconstruction image can be obtained by 3 -d construction reconstruction theory.

Further research would focus on two aspects of algorithm optimization and stereo vision optimization.

\section{ACKNOWLEDGMENT}

This paper is supported by Wuhan Planning Project of Science and Technology. (Grant No. 201051099415-11)

\section{REFERENCES}

[1] Roy-Chowdhury, A.K. Chellappa, R. Statistical bias in 3- D reconstruction from a monocular video. IEEE trans. Image Processing.8(14):1057-1062.Aug.2005.

[2] Saxena, A. Min Sun Ng, A.Y. 3-D Reconstruction from Sparse Views using Monocular Vision. IEEE trans. Computer Vision, 2007.12(26):18.Oct.2007.

[3] Han, Y. Geometric algorithms for least squares estimation of 3-D information from monocular image. IEEE trans. on Circuis and Systems for Video Technology. 2(15):269-282.

[4] Woodward, A. Da An Gimel'farb, G. Delmas, P. A Comparison of Three 3-D Facial Reconstruction Approaches. IEEE trans. Multimedia and Expo, 2006 IEEE International Conference on. 2057 - 2060. July 2006.

[5] Ostermann, J. 3D information coding. .IEEE trans. Picture Coding Symposium (PCS), 2010.9(8):19.Dec.2010.

[6] Jiang Ze-tao. Zheng Bi-na .Wu Min. Wu Wen-huan. A Fully Automatic 3D Reconstruction Method Based on Images. IEEE trans. Computer Science and Information Engineering, 2009 WRI World Congress on.7(24):327-331.April.2009.

[7] Chang Li. Yong Zhou.3D auto-reconstruction for street elevation based on line and plane feature. IEEE trans. Computer and Automation Engineering (ICCAE), 2010 The 2nd International Conference on. 3(1):460-466.April.2010.

[8] Kuo Zhang Qingchang Tan Chunyan Wang Qichao Li .3D reconstruction of surface topography based on 2D Fourier Transform. IEEE trans.Computer Mechatronics, Control and Electronic Engineering (CMCE), 2010 International Conference on (CMCE)..8(2):49-52.Aug.2010. 
TAB LE I. SEQUENCE OF LEFT PATH DATA

\begin{tabular}{|c|c|c|c|c|c|c|c|c|c|c|c|}
\hline $\mathrm{yL}$ & 0 & 1 & 2 & 3 & 4 & 5 & 6 & $\ldots \ldots$ & 771 & 772 & 773 \\
\hline $\mathrm{xL}$ & 470 & 470 & 470 & 470 & 470 & 471 & 470 & $\ldots \ldots$ & 589 & 589 & 588 \\
\hline $\mathrm{yR}$ & 0 & 1 & 2 & 3 & 4 & 5 & 6 & $\ldots \ldots$ & 771 & 772 & 773 \\
\hline $\mathrm{xR}$ & 762 & 762 & 761 & 761 & 761 & 760 & 760 & $\ldots \ldots$ & 567 & 567 & 566 \\
\hline
\end{tabular}

